\title{
ON THE COMMUTATIVITY OF SEMI-PRIME RINGS
}

\author{
ABDULLAH H. AL-MOAJIL
}

(Received 23 July 1980)

Communicated by R. Lidl

\begin{abstract}
It is shown that if $R$ is a 2-torsion-free semi-prime ring such that $[x y,[x y, y x]]=0$ for all $x, y \in R$, then $R$ is commutative.

1980 Mathematics subject classification (Amer. Math. Soc.): primary 16 A 38, 16 A 70; secondary 16 A 12.

Key words and phrases: nilpotent, nilpotent ideal, semi-prime.

A commutativity theorem which was proven by Gupta [2] asserts that a division ring $D$ which satisfies the polynomial identity $x y^{2} x=y x^{2} y$ for all $x, y \in D$ must be commutative. This was generalized by Awtar [1] who proves that if $R$ is a semi-prime ring (that it contains no non-zero nilpotent ideals) and if $x y^{2} x-$ $y x^{2} y$ is central for all $x, y \in R$, then $R$ is commutative. In this paper we give a further generalization of this result for the case of 2-torsion-free rings. We will prove the following theorem.
\end{abstract}

THEOREM. Let $R$ be a 2-torsion-free semi-prime ring. If $x y$ commutes with $x y^{2} x-y x^{2} y$ for all $x, y \in R$ then $R$ is commutative.

The proof we give is elementary and does not make use of any of the previously known commutativity theorems. First we need to prove some lemmas. We will let $[x, y]$ denote $x y-y x$ as usual.

(C) Copyright Australian Mathematical Society 1982 
LEMMA 1. If $R$ is $a$ ring and $x, y \in R$ satisfy $[x,[x, y]]=0$ then $\left[x^{2}, y\right]=$ $2 x[x, y]$.

Proof. We have $\left[x^{2}, y\right]-2 x[x, y]=-y x^{2}-x^{2} y+2 x y x=[x,[x, y]]=0$.

LEMMA 2. If $x, y$ satisfy the hypothesis of Lemma 1 then $\left[x,\left[x, y^{2}\right]\right]=2[x, y]^{2}$.

Proof. We have

$$
\begin{aligned}
{\left[x,\left[x, y^{2}\right]\right]-2[x, y]^{2} } & =x^{2} y^{2}+y^{2} x^{2}-2(x y)^{2}-2(y x)^{2}+2 y x x^{2} \\
& =\left(x^{2} y+y x^{2}-2 x y x\right) y+y\left(x^{2} y+y x^{2}-2 x y x\right) \\
& =[x,[x, y]] y+y[x,[x, y]]=0 .
\end{aligned}
$$

LEMMA 3. Let $R$ be a ring satisfying the identity $[x y,[x y, y x]]=0$ for all $x, y \in R$. If there exists a non-zero element $x \in R$ such that $x^{2}=0$ then $R$ is not semi-prime.

Proof. Let $y$ be an arbitrary element of $R$. Applying the identity above to $x$ and $y-y x$, and using the fact that $x^{2}=0$ we get

$$
\begin{aligned}
0 & =[x(y-y x),[x(y-y x),(y-y x) x]]=[x(y-y x),[x(y-y x), y x]] \\
& =\left[x(y-y x), x y^{2} x-(x y)^{2} x\right]=(x y)^{2} y x-(x y)^{3} x .
\end{aligned}
$$

Therefore,

$$
(x y)^{2} y x=(x y)^{3} x
$$

Now apply the identity to $x y x$ and $y$ to get

$$
\begin{aligned}
0 & =[x y x y,[x y x y, y x y x]] \\
& =\left[x y x y, x y x y^{2} x y x\right]=(x y)^{4}(y x)^{2} .
\end{aligned}
$$

Using (1) to substitute $(x y)^{3} x$ for $(x y)^{2} y x$ in (2), we obtain $(x y)^{6} x=0$. Therefore, $(x y)^{7}=0$.

Since $y$ was arbitrary this proves that $z^{7}=0$ for all $z$ in the right ideal $x R$. Therefore, it follows by [4, Lemma 1.1], that $R$ contains a non-zero nilpotent ideal.

Proof of The Theorem. Since $R$ is semi-prime we may assume, in view of Lemma 3, that $R$ contains no nilpotent elements. Let $x, y \in R$ be arbitrary. Then $[x y,[x y, y x]]=0$ by assumption. This obviously implies that 
$\left[(x y)^{2},[x y, y x]\right]=0$. Moreover, by Lemma $1,\left[(x y)^{2}, y x\right]=2 x y[x y, y x]$. Therefore, $(x y)^{2}$ commutes with $\left[(x y)^{2}, y x\right]$. That is,

$$
\left[(x y)^{2},\left[(x y)^{2}, y x\right]\right]=0 .
$$

Using (3) and Lemma 2 we get,

$$
\begin{aligned}
2\left[(x y)^{2}, y x\right]^{2} & =\left[(x y)^{2},\left[(x y)^{2},(y x)^{2}\right]\right] \\
& =[(x y x) y,[(x y x) y, y(x y x)]]=0
\end{aligned}
$$

by taking $z=x y x$ and applying the assumption on elements of $R$.

Since $R$ is 2-torsion-free and contains no nilpotent elements this implies that $\left[(x y)^{2}, y x\right]=0$. Therefore, since $[y x,[y x, x y]]=0$, Lemma 2 implies that $2[y x, x y]^{2}=[y x, 0]=0$. Hence, by the assumption on $R,[y x, x y]=0$, that is

$$
x y^{2} x=y x^{2} y .
$$

Since $x$ and $y$ were arbitrary, this holds for all $x, y \in R$. Therefore, replacing $y$ with $x+y$ in (4) we get $x^{2} y x+x y x^{2}=x^{3} y+y x^{3}$, that is

$$
\left[x^{2},[x, y]\right]=0 \text {. }
$$

Since $\left[x^{2}, y\right]=x[x, y]+[x, y] x$ and $x^{2}$ commutes with $[x, y]$ by (5), we get $\left[x^{2},\left[x^{2}, y\right]\right]=0$. Moreover, replacing $y$ with $y^{2}$ we obtain $\left[x^{2},\left[x^{2}, y^{2}\right]\right]=0$. Hence, by Lemma $2,2\left[x^{2}, y\right]^{2}=\left[x^{2},\left[x^{2}, y^{2}\right]\right]=0$, which implies that $\left[x^{2}, y\right]=0$ or

$$
x^{2} y=y x^{2} .
$$

Now replacing $y$ with $x^{2}+y$ in (4) we obtain $\left[x^{3},[x, y]\right]=0$ which implies that $\left[x^{3},\left[x^{3}, y\right]\right]=0$, since $\left[x^{3}, y\right]=x^{2}[x, y]+x[x, y] x+[x, y] x^{2}$. Repeating the argument above for $x^{3}$ and $y^{2}$ we obtain,

$$
x^{3} y=y x^{3} .
$$

Applying (6) and (7) we get $\left(x y x-x^{2} y\right)^{2}=0$. Thus $x y x=x^{2} y=y x^{2}$. Replacing $y$ with $y^{2}$ we get $x y^{2} x=x^{2} y^{2}=y^{2} x^{2}$. Therefore, $(x y-y x)^{2}=0$ which implies that $x y=y x$. Since $x$ and $y$ were arbitrary we conclude that $R$ is commutative.

At the end we point out that one could have quoted Gupta's result [2] after equation (4) or Herstein's theorem [5] after equation (6) to conclude the proof. This would have been on the expense of the self-containment of this paper. Moreover, the part of the proof that starts after (4) gives an alternative proof to Gupta's theorem. 


\section{References}

[1] R. Awtar, 'A remark on the commutativity of certain rings', Proc. Amer. Math. Soc. 41 1973, 370-372.

[2] R. Gupta, 'Nilpotent matrices with invertible transpose', Proc. Amer. Math. Soc. 24 1970, 572-575.

[3] I. Herstein, Non-commutative rings, (Carus Monograph 16, Mathematical Association of America, Washington D.C., 1968).

[4] I. Herstein, Topics in ring theory, (University of Chicago Press, 1969).

[5] I. Herstein, 'A commutativity theorem', J Algebra 38 1976, 112-118.

[6] N. Jacobson, Structure of rings, (Amer. Math. Soc. Colloquium Publications 37, Amer. Math. Soc., Providence, R.I., 1964).

Gulf Organization for Industrial Consulting

P. O. Box 5114

Doha, Quatar 